OPEN ACCESS

Edited by:

Sei Saitoh,

Fujita Health University, Japan

Reviewed by: Chihiro Hama, Kyoto Sangyo University, Japan Yuji Hara,

University of Shizuoka, Japan Kazuhiko Kume, Nagoya City University, Japan

${ }^{*}$ Correspondence: Shoko Nishihara shoko@soka.ac.jp

Received: 30 August 2021 Accepted: 29 September 2021 Published: 18 October 2021

Citation: Itoh $K$ and Nishihara S (2021) Mucin-Type O-Glycosylation in the Drosophila Nervous System. Front. Neuroanat. 15:767126. doi: 10.3389/fnana.2021.767126

\section{Mucin-Type O-Glycosylation in the Drosophila Nervous System}

\author{
Kazuyoshi Itoh ${ }^{1}$ and Shoko Nishihara ${ }^{1,2 *}$ \\ ${ }^{1}$ Glycan \& Life Systems Integration Center (GaLSIC), Soka University, Hachioji, Japan, ${ }^{2}$ Department of Biosciences, Graduate \\ School of Science and Engineering, Soka University, Hachioji, Japan
}

Mucin-type O-glycosylation, a predominant type of $O$-glycosylation, is an evolutionarily conserved posttranslational modification in animals. Mucin-type O-glycans are often found on mucins in the mucous membranes of the digestive tract. These glycan structures are also expressed in other cell types, such as blood cells and nephrocytes, and have crucial physiological functions. Altered expression of mucin-type O-glycans is known to be associated with several human disorders, including Tn syndrome and cancer; however, the physiological roles of mucin-type O-glycans in the mammalian brain remains largely unknown. The functions of mucin-type O-glycans have been studied in the fruit fly, Drosophila melanogaster. The basic structures of mucin-type O-glycans, including Tn antigen (GalNAca1-Ser/Thr) and T antigen (Galß1-3GalNAco1-Ser/Thr), as well as the glycosyltransferases that synthesize them, are conserved between Drosophila and mammals. These mucin-type O-glycans are expressed in the Drosophila nervous system, including the central nervous system (CNS) and neuromuscular junctions (NMJs). In primary cultured neurons of Drosophila, mucin-type O-glycans show a characteristic localization pattern in axons. Phenotypic analyses using mutants of glycosyltransferase genes have revealed that mucin-type O-glycans are required for CNS development, NMJ morphogenesis, and synaptic functions of NMJs in Drosophila. In this review, we describe the roles of mucin-type O-glycans in the Drosophila nervous system. These findings will provide insight into the functions of mucin-type O-glycans in the mammalian brain.

\footnotetext{
Keywords: mucin-type O-glycans, mucin-type O-glycosylation, $\mathrm{T}$ antigen, Drosophila, nervous system, neuromuscular junction
}

\section{INTRODUCTION}

Mucin-type $O$-glycans, one of the major types of $O$-glycan, are evolutionarily conserved in animals. They are generally found on mucins, which are mucus glycoproteins expressed on the mucous membranes of the digestive tract, and are necessary for protecting the gut, for example, from physical damage and bacterial infection. Mucin-type $O$-glycans are conjugated to many proteins other than mucins and have been shown to play crucial roles in various mammalian cell types, such as blood cells (Wang et al., 2012; Kudo et al., 2013), nephrocytes (Fuseya et al., 2020; Stotter et al., 2020), and submandibular cells (Tian et al., 2012). In addition, unusual expression of mucin-type O-glycans has been associated with several human disorders, including Tn syndrome (Berger, 1999; Ju and Cummings, 2005), IgA nephropathy (Suzuki et al., 2008; Hiki, 2009), heterotaxy (Fakhro et al., 2011; Boskovski et al., 2013), and cancer (Springer, 1984; Ju et al., 2008; Radhakrishnan et al., 2014). However, the functions of mucin-type $O$-glycans in the mammalian brain remains unclear. 
In contrast to mammals, the functions of mucin-type $O$ glycans have been well studied in the Drosophila nervous system. The genome of Drosophila, which is used as a model organism, is about $60 \%$ homologous to that of humans. Notably, about $75 \%$ of the genes responsible for human diseases are present in Drosophila as orthologs (Ugur et al., 2016). About half of the genes encoding human glycosyltransferases have orthologs in Drosophila (Yamamoto-Hino et al., 2015), and those orthologs have been shown to synthesize various glycan structures that are conserved in mammals, including some types of mucin-type O-glycan such as Tn antigen (GalNAca1-Ser/Thr) and $\mathrm{T}$ antigen (Gal $\beta 1$-3GalNAc $\alpha 1-S e r / T h r ; ~ N i s h i h a r a, 2020)$. Here, we summarize our current understanding of the roles of mucin-type $O$-glycans in the Drosophila nervous system.

\section{Biosynthesis of Mucin-Type O-Glycans in Drosophila}

In mammals, there are various structures of mucin-type $O$ glycans, including four common core structures (Bennett et al., 2012). In Drosophila, by contrast, there are three main glycan structures of mucin-type $O$-glycans, namely, Tn antigen, $\mathrm{T}$ antigen (core 1), and glucuronylated T antigen (GlcA $\beta 1-3 \mathrm{Gal} \beta 1$ 3GalNAca1-Ser/Thr; Figure 2A; Kramerov et al., 1996; Aoki et al., 2008; Breloy et al., 2008). Mass spectrometry analysis revealed that the expression levels of $\mathrm{Tn}$ antigen, $\mathrm{T}$ antigen, and glucuronylated $\mathrm{T}$ antigen account for, respectively, about $20 \%, 55 \%$, and $10 \%$ of O-glycans in Drosophila embryos (Aoki et al., 2008). In mammals and Drosophila, polypeptide $N$ acetylgalactosaminyl-transferases (ppGalNAcTs) synthesize Tn antigen by the addition of an $\mathrm{N}$-acetylgalactosamine (GalNAc) to serine (Ser) or threonine (Thr) residues of the core protein (Bennett et al., 2012; Tran and Hagen, 2013). Humans and mice have 20 and 19 ppGalNAcTs, respectively, whereas Drosophila has 12 ppGalNAcTs.

After the addition of GalNAc, core $1 \beta 1$, 3-galactosyltransferase 1 (C1GalT1) transfers galactose (Gal) to the GalNAc residue in a $\beta 1$, 3-linkage and synthesizes $\mathrm{T}$ antigen both in mammals and in Drosophila. Whereas mammalian C1GalT1 requires a molecular chaperone, Cosmc (also known as C1GalT1C1), for its enzymatic activity (Ju and Cummings, 2002), Drosophila C1GalT1 (dC1GalT1) does not (Müller et al., 2005). After the synthesis of $\mathrm{T}$ antigen, sialylated $\mathrm{T}$ antigen (Sia $\alpha 2-3 \mathrm{Gal} \beta 1-3 \mathrm{GalNAc} \alpha 1-\mathrm{Ser} / \mathrm{Thr}$ ) is synthesized in mammals; however, sialylated $\mathrm{T}$ antigen has not been identified in Drosophila (Schwientek et al., 2007; Aoki et al., 2008). In addition, although one sialyltranseferase (SiaT) has been identified in Drosophila, it does not show catalytic activity for the transfer of sialic acid (Sia) to $\mathrm{T}$ antigen (Koles et al., 2004). Instead of the synthesis of sialylated $\mathrm{T}$ antigen in Drosophila, glucuronylated $\mathrm{T}$ antigen is produced by $\beta 1$, 3-glucuronyltransferase-P (dGlcAT-P), which predominantly transfers glucuronic acid (GlcA) to the Gal residue in a $\beta 1$, 3-linkage (Kim et al., 2003; Breloy et al., 2016; Itoh et al., 2018). Therefore, Drosophila glucuronylated T antigen is considered to correspond to mammalian sialylated $\mathrm{T}$ antigen because the two glycan structures contain negatively charged monosaccharides, namely, GlcA and Sia.

\section{Expression of Mucin-Type O-Glycans in the Drosophila Nervous System} In vivo Expression of Mucin-Type O-Glycans

In Drosophila embryos, dC1GalT1 mRNA and T antigen, which can be labeled by anti-T antigen antibody or peanut agglutinin (PNA), are expressed in the central nervous system (CNS), including the brain and ventral nerve cord (VNC; Tian and Ten Hagen, 2007; Lin et al., 2008; Yoshida et al., 2008). T antigen is first expressed in the CNS at embryonic stage 13 and then abundantly expressed in the ladder-like pattern of the VNC at stage 16 (Yoshida et al., 2008). T antigen colocalizes with antigen of the CNS marker BP102. In dC1GalT1 mutant embryos, T antigen expression in the CNS is completely abolished, showing that dC1GalT1 has a central role in T antigen synthesis in the CNS during embryonic development (Lin et al., 2008; Yoshida et al., 2008).

At the larval stages, dC1GalT1 mRNA is also detected in the CNS (Lin et al., 2008). Moreover, lectin staining has revealed that both $\mathrm{Tn}$ antigen and $\mathrm{T}$ antigen are expressed in larval neuromuscular junctions (NMJs; Haines et al., 2007; Dani et al., 2014; Jumbo-Lucioni et al., 2014; Itoh et al., 2016, 2018). In dC1GalT1 mutant larvae, $\mathrm{T}$ antigen expression at NMJs is decreased, showing that dC1GalT1 is required for $\mathrm{T}$ antigen synthesis at these synapses (Itoh et al., 2016). Moreover, T antigen expression at NMJs is upregulated in $d G l c A T-P$ mutants, suggesting that glucuronylated $\mathrm{T}$ antigen is also expressed at these synapses (Figure 2B; Itoh et al., 2018).

Previous studies have revealed that laminin subunits and dystroglycan $(\mathrm{Dg})$, both of which are expressed in the Drosophila nervous system, carry mucin-type $O$-glycans (Haines et al., 2007; Bogdanik et al., 2008; Lin et al., 2008; Schneider and Baumgartner, 2008; Nakamura et al., 2010); however, the functions of mucin-type $O$-glycans on these core proteins remain unknown. Dg also carries $O$-mannosyl glycans, which are synthesized by the Drosophila protein $O$-mannosyltransferase 1 (dPOMT1) and dPOMT2 (Ichimiya et al., 2004; Ueyama et al., 2010). Loss of either of these two enzymes causes defects in NMJs and axonal connections of sensory neurons (Wairkar et al., 2008; Baker et al., 2018).

\section{Localization of Mucin-Type O-Glycans in Primary Cultured Neurons}

It has been reported that $\mathrm{T}$ antigen shows distinctive localization in the axons of primary cultured neurons derived from Drosophila embryos (Kinoshita et al., 2017). The axons of primary cultured neurons are divided into two compartments, namely, the proximal segment and the distal segment (Figure 1A; Katsuki et al., 2009). Both the axon guidance receptors, Derailed (DRL) and BP102 antigen are specifically localized to the proximal segment of the axon, while two other axon guidance receptors, roundabout $2(\mathrm{ROBO} 2)$ and $\mathrm{ROBO} 3$, are specifically localized to the distal segment of the axon. $\mathrm{T}$ antigen localization is also restricted to the proximal segment of axons, similar to DRL and BP102 antigen (Kinoshita et al., 2017).

Ultrastructural analysis by atmospheric scanning electron microscopy (ASEM) has revealed that $\mathrm{T}$ antigen accumulates near the boundary between the proximal and distal segments of 


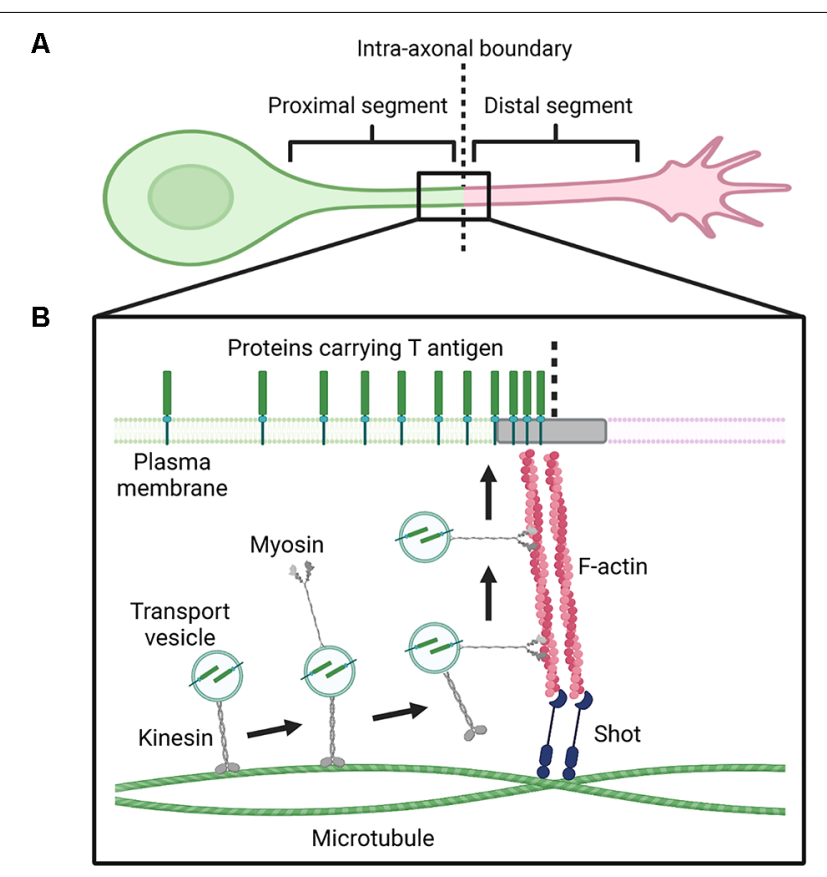

FIGURE 1 | Proposed mechanism of Shot-mediated transport of membrane proteins carrying mucin-type core 1 glycans to the proximal axonal segment. (A) Schematic diagram showing a Drosophila primary cultured neuron, in which the axon is divided into proximal and distal segments by the intra-axonal boundary. (B) Schematic diagram showing the proposed transport mechanism. Transport vesicles containing proteins carrying $T$ antigen are transferred by kinesin on microtubules from the soma to the point where two tubulin bundles are in contact with each other in the axon. The vesicles become detached from the microtubules and are transferred to F-actin, which is linked to microtubules by the crosslinking protein Shot. Subsequently, the vesicles are transferred by myosin on F-actin to the plasma membrane at the intra-axonal boundary, where the membrane proteins carrying $T$ antigen spread to the proximal segment. Figure created with BioRender (https://BioRender.com).

the axon, known as the intra-axonal boundary. Null mutations of the gene encoding Short stop (Shot), a protein that crosslinks F-actin and microtubules, resulting in impairment of the proximal localization of $\mathrm{T}$ antigen, which in turn is rescued by the expression of wild-type Shot. ASEM analysis has revealed that the formation of microtubule bundles is disturbed in Shot null mutants, showing that Shot is involved in microtubule formation. Moreover, loss of the F-actin-binding domain of Shot also results in impairment of the proximal localization of $\mathrm{T}$ antigen but does not affect microtubule bundles. Therefore, these results demonstrate that the F-actinbiding domain of Shot is essential for the trafficking of mucin-type core 1 glycans to the proximal axonal segment. ASEM observations have also revealed that F-actin accumulates near the intra-axonal boundary similar to $\mathrm{T}$ antigen (Kinoshita et al., 2014). Furthermore, two tubulin bundles in the axon are in contact at the intra-axonal boundary. Taking all these findings together, Kinoshita et al. (2017) have proposed a mechanism for Shot-mediated axonal trafficking of membrane proteins carrying mucin-type core 1 glycans to the proximal axonal segment (Figure 1B).

\section{Functions of Mucin-Type O-Glycans in the Central and Peripheral Nervous System}

The phenotypes of the CNS in $d C 1$ GalT1 and dGlcAT-P mutants have been analyzed. Although depletion of dC1GalT1 leads to loss of $\mathrm{T}$ antigen expression in the embryonic CNS, the morphology of the CNS is normal (Lin et al., 2008; Yoshida et al., 2008). These data show that $\mathrm{T}$ antigen is dispensable for the development of the CNS during embryogenesis. In the larval stages, however, dC1GalT1 null mutants display a malformed brain hemisphere and greatly extended VNC (Lin et al., 2008), showing that mucin-type core 1 glycans are required for subsequent CNS development. Moreover, this extended VNC phenotype is also observed in $d G l c A T-P$ null mutants (Pandey et al., 2011). The $d G l c A T-P$ mutant larvae also display a reduction in the length of motor neuron axons that extend from the VNC to target muscles. Because the flies grow extensively during larval stages, motor neuron axons need to extend in parallel to the enlargement of the larval body. Pandey et al. (2011) have therefore suggested that, in $d G l c A T-P$ mutants, the VNC extends abnormally in order to compensate for the tension caused by the impaired growth of motor neuron axons. The extended VNC phenotype is rescued by overexpressing dGlcAT-P in hemocytes. Collectively, these data suggest the possibility that blood cell-derived mucin-type $O$-glycans produced by dGlcAT-P are involved in the elongation of peripheral nerves.

\section{Functions of Mucin-Type $\mathbf{O}$-Glycans in Neuromuscular Junctions \\ The Drosophila Neuromuscular Junction as a Model System for Mammalian Central Synapses}

Drosophila larval NMJs are a good model for studying synapses in the mammalian CNS (Menon et al., 2013). Drosophila NMJs are easy to observe because the synapses are large and specified individually. They are glutamatergic and use ionotropic glutamate receptors (GluRs) that are homologous to AMPA-type GluRs in the mammalian CNS. In Drosophila larvae, motor neuron axons stereotypically innervate postsynaptic muscle cells, which are respectively numbered and regularly arranged in each hemisegment (Figure 2C). The NMJs consist of a branched chain of synaptic boutons, which are oval-shaped structures. Each presynaptic bouton is surrounded by subsynaptic reticulum, which is a folded structure of the postsynaptic membrane. The boutons contain an active zone, which is a site of neurotransmitter release, on the presynaptic side and GluR clusters on the postsynaptic side.

\section{Phenotypic Analysis of ppGalNAcT Mutants}

The functions of mucin-type O-glycans in NMJs have been studied in Drosophila larvae. Loss of either ppGalNAcT3 or ppGalNAcT35A upregulates (i) expression of Tn antigen; (ii) molecular assemblies of presynaptic active zones and postsynaptic GluRs; and (iii) neurotransmission strength evoked at the NMJs (Dani et al., 2014). Ultrastructural observation has shown that the number of presynaptic vesicles near the active zone and the depth of the postsynaptic pocket are increased in the two ppGalNAcT mutants. In addition, the components of integrin signaling, including the synaptic Position Specific 2 
A

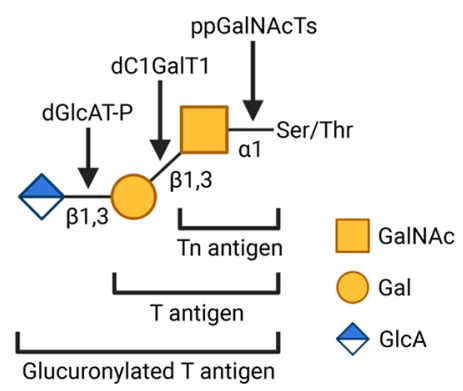

B

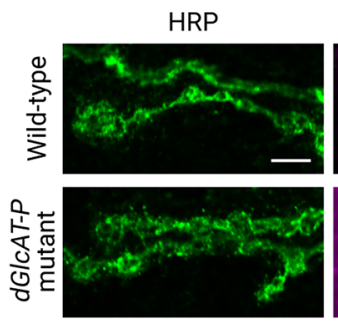

PNA
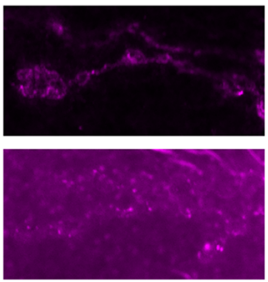
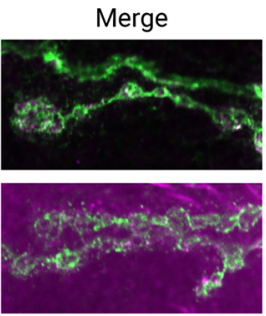

C

\section{D}

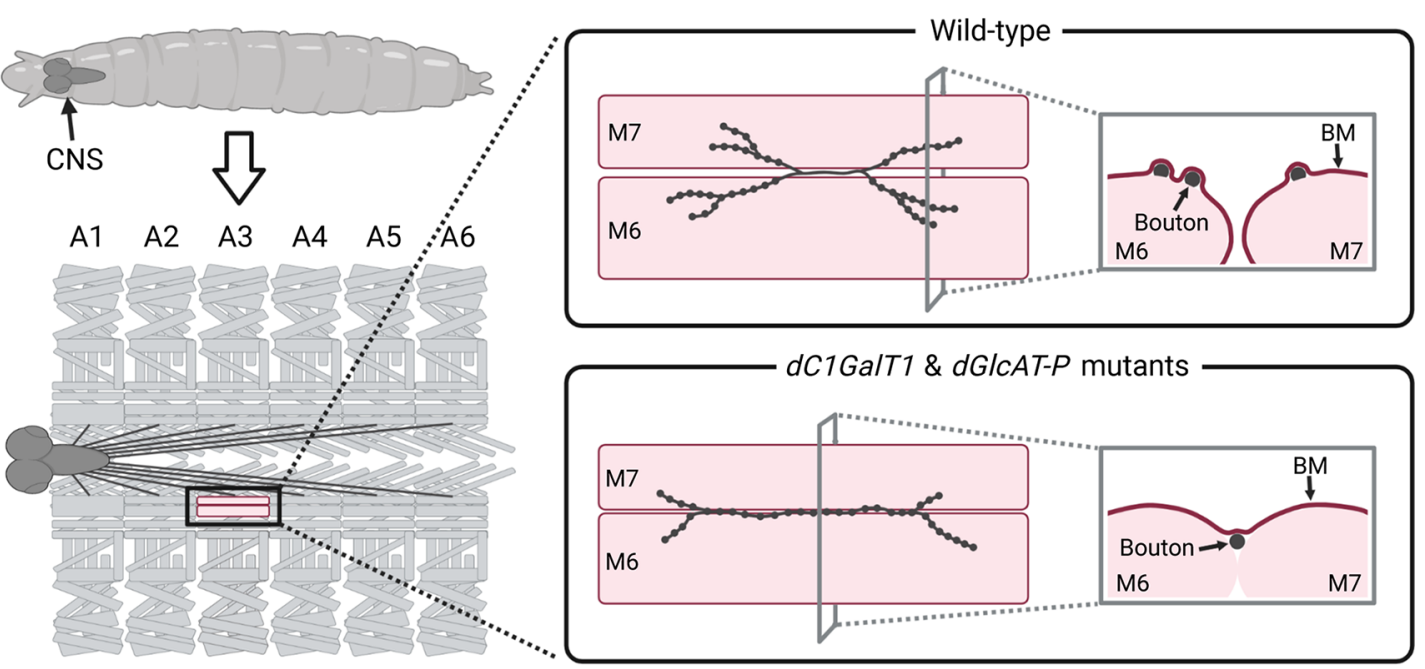

E
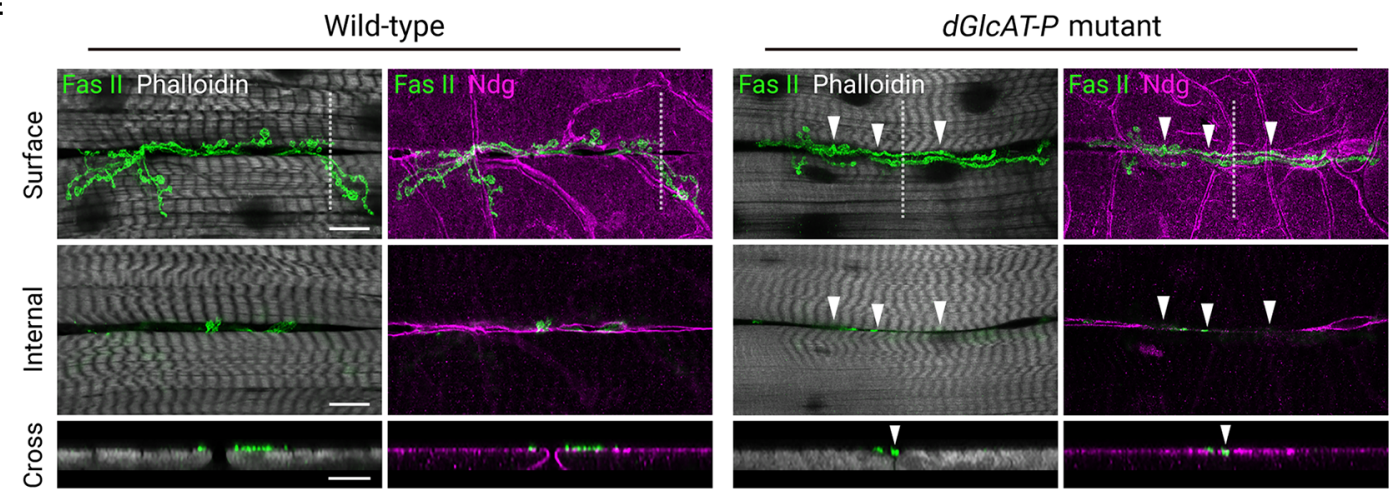

F

Wild-type
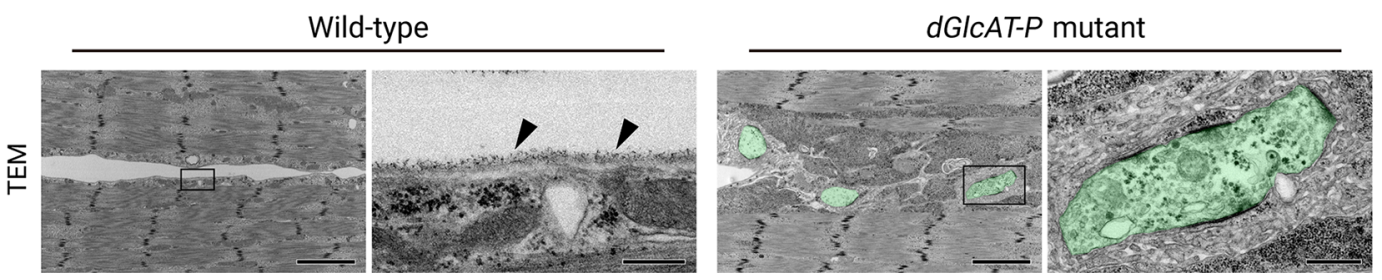

FIGURE 2 | Mislocalization of NMJ boutons and loss of BM components in dC1GalT1 and dG/cAT-P mutants. (A) In Drosophila, there are three main glycan structures of mucin-type O-glycans, namely, Tn antigen (GalNAca1-Ser/Thr), T antigen (Galß1-3GalNAca1-Ser/Thr), and glucuronylated T antigen

(GlcAB1-3Galß1-3GalNAca1-Ser/Thr). Polypeptide N-acetylgalactosaminyl-transferases (ppGalNAcTs) transfer N-acetylgalactosamine (GalNAc) to serine (Ser) or threonine (Thr) residues of the core protein in an $\alpha 1$-linkage to synthesize Tn antigen. Core $1 \beta 1,3$-galactosyltransferase 1 (dC1GalT1) transfers galactose (Gal) to the GalNAc residue of Tn antigen in a $\beta 1,3$-linkage to synthesize T antigen. $\beta 1,3$-glucuronyltransferase-P (dGlcAT-P) transfers glucuronic acid (GlcA) to the Gal residue of T antigen in a $\beta 1,3$-linkage to synthesize glucuronylated T antigen. (B) Confocal images of larval neuromuscular junctions (NMJs) on muscle 6 in wild-type (upper) and dGICAT-P mutant (lower). NMJs are stained with anti-horseradish peroxidase (HRP) antibody (a presynaptic marker) and peanut agglutinin (PNA; a T antigen 


\section{FIGURE 2 | Continued}

marker). T antigen expression colocalizes with the presynaptic marker. The expression level of T antigen at NMJs, as well as on the muscle surface around them, is higher in dG/CAT-P mutant than in wild-type. Scale bar: 5 $\mu \mathrm{m}$. (C) Upper, schematic diagram showing Drosophila larva. CNS, central nervous system. Lower, schematic diagram showing structures of the nervous system and body wall muscles of a larva, dissected along the dorsal midline. Motor neuron axons extend from the CNS to each abdominal segment, which are numbered in A1-A6. Muscle cells in each hemisegment are regularly arranged, and their arrangement is symmetric. Muscles 6 and 7 in A3 are indicated in magenta. (D) Left, whereas NMJ boutons are localized near the boundary between muscles 6 and 7 in wild-type larvae, many NMJ boutons are mislocalized at the muscle $6 / 7$ boundary in $d C 1$ GalT1 and dGICAT-P mutants. The mutant NMJs also show a decreased number of branches. Right, cross-sectional view of muscles 6 and 7 . Whereas the two muscles are apart from each other and individually covered with separate basement membranes (BMs) in wild-type, the two muscles are connected through the mislocalized boutons and covered with a single continuous BM in $d C 1 \mathrm{Gal} / \mathrm{T}$ and $d \mathrm{G} / \mathrm{CAT}-\mathrm{P}$ mutants. The components of BMs, such as type IV collagen and nidogen (Ndg), are lost at or just beneath the mislocalized boutons. M6, muscle 6; M7, muscle 7. Figure created with BioRender (https://BioRender.com). (E) Confocal images of NMJs on muscles 6 and 7 in A3 in wild-type (left) and dG/cAT-P mutant (right). NMJs, muscle fibers, and BMs are stained with anti-fasciclin II (Fas II) antibody, Phalloidin, and anti-Ndg antibody, respectively. Upper, in the surface sectional view of the muscles, NMJ boutons are localized near the boundary between the two muscles in wild-type, but are mislocalized at the boundary in dGICAT-P mutant (arrowheads). Middle, in the internal sectional view of the muscles, each muscle is covered with a continuous BM at the boundary in wild-type, but the BMs are partially lost at the boundary in dGICAT-P mutant (arrowheads). Lower, in the cross-sectional view of the areas within white dotted lines in the upper panels, the two muscles are apart from each other and are individually covered with separate BMs in wild-type, but are attached to each other and covered with a single continuous BM in dG/cAT-P mutant. A bouton is ectopically localized at the muscle $6 / 7$ boundary in $d G / c A T-P$ mutant (arrowhead). Scale bars: $20 \mu \mathrm{m}$ (upper and middle panels) and 10 $\mu \mathrm{m}$ (lower panel). (F) Transmission electron micrographs (TEM) of the muscle 6/7 boundary in wild-type and dG/CAT-P mutant. For each genotype, the right panel shows a high magnification view of the area bordered by the rectangle in the left panel. In wild-type, arrowheads indicate a BM that covers the muscle surface. In $d G / c A T-P$ mutant, the presynaptic side of mislocalized NMJ boutons is indicated in green. Whereas no NMJ boutons are observed in the cleft between the two muscles in wild-type, some NMJ boutons are observed in the cleft and connect the two muscles in dGIcAT-P mutant. Scale bars: $3 \mu \mathrm{m}$ (left panels), $300 \mathrm{~nm}$ (right panel, wild-type), and $500 \mathrm{~nm}$ (right panel, $d G / c A T-P$ mutant).

( $\alpha$ PS2) integrin receptor and transmembrane tenascin ligand, are downregulated at the NMJs of both ppGalNAcT mutants. Moreover, the two mutants display the impairment of activitydependent synaptic plasticity and the suppression of activitydependent changes in integrin signaling and postsynaptic pocket size. All these phenotypes are restored to wild-type in double mutants of the two ppGalNAcTs. Tissue-specific rescue experiments have revealed that, on both the pre- and postsynaptic side of NMJs, ppGalNAcT3 and ppGalNAcT35A regulate Tn antigen expression, synaptic molecular assemblies, neurotransmission strength, and $\alpha$ PS2 integrin expression. Furthermore, inhibition of integrin signaling blocks synaptic plasticity in the two ppGalNAcT mutants.

Collectively, therefore, Dani et al. (2014) have suggested that ppGalNAcT3 and ppGalNAcT35A genetically suppress each other on both sides of the synapse to regulate $O$-GalNAc glycosylation, as well as synaptic molecular assemblies, neurotransmission strength, and activity-dependent plasticity through integrin signaling. The suppressive regulation between $p p G a l N A c T 3$ and ppGalNAcT35A is apparently linked to the balanced function of the two genes. As a result, the loss of either ppGalNAcT3 or ppGalNAcT35A impairs the balance between the two genes; thus, other $p p G a l N A c T$ may become dysregulated, leading to the upregulation of Tn antigen expression and other phenotypes as described above. Because of the suppressive interaction between ppGalNAcT3 and ppGalNAcT35A, these phenotypes are restored to wild-type in double mutants.

\section{Phenotypic Analysis of $d C 1 G a / T 1$ and $d G / c A T-P$ Mutants}

Recent studies have found that mucin-type O-glycans synthesized by dC1GalT1 and dGlcAT-P also play crucial roles in larval NMJs. Mutation in either $d C 1$ GalT1 or $d G l c A T-P$ leads to morphological defects in the NMJs formed on the large abdominal muscles 6 and 7. In Drosophila, the axon terminal of a motor neuron branches and establishes NMJ boutons near the boundary between muscles 6 and 7. While most boutons are localized near the muscle 6/7 boundary in wild-types, many NMJ boutons are ectopically localized at the boundary in $d C 1$ GalT1 and $d G l c A T-P$ mutants (Figures 2D,E; Itoh et al., 2016, 2018).

In Drosophila, basement membranes (BMs) cover the surfaces of the muscles and NMJ boutons, except for the synaptic cleft between the presynaptic bouton and the postsynaptic muscle (Koper et al., 2012). In the internal sectional view of muscles 6 and 7, wild-type shows continuous BMs at the muscle 6/7 boundary; however, dC1GalT1 and dGlcAT-P mutants display a partial loss of BM components, such as type IV collagen and nidogen $(\mathrm{Ndg}$ ), at the muscle $6 / 7$ boundary (Figure 2E; Itoh et al., 2016, 2018). In the cross-sectional view of the two muscles, they are individually covered with separate BMs in wild-type; however, in the $d C 1 G a l T 1$ and $d G l c A T-P$ mutants, they are covered with a single continuous $\mathrm{BM}$, and the $\mathrm{BM}$ components are lost at or just beneath the mislocalized bouton at the muscle 6/7 boundary (Figures 2D,E). Further analyses have revealed that the mislocalized boutons in the two mutants tend to localize at or just above the site of the missing BM components at the muscle $6 / 7$ boundary. Ultrastructural analysis has further revealed that some boutons are localized at the cleft between muscles 6 and 7 and connect these two muscles (Figure 2F). During larval stages, BM components such as type IV collagen are secreted from the fat body to hemolymph (body fluid) and deposited on various tissue surfaces in contact with hemolymph (Pastor-Pareja and $\mathrm{Xu}, 2011$ ). It is possible that, in the $d C 1 G a l T 1$ and $d G l c A T-P$ mutants, the mislocalized boutons, which connect the two muscles, physically prevent the deposition of BM components just below them, leading to the formation of a single continuous BM across the two muscles. Therefore, the ectopic localization of NMJ boutons at the muscle $6 / 7$ boundary may be a direct cause of the loss of BM components just beneath these boutons. In addition, analysis of double heterozygous mutants of $d C 1 \mathrm{GalT} 1$ and $d G l c A T-P$ reveals that the two genes genetically interact with each other. Taken together, these data clearly demonstrate that glucuronylated $\mathrm{T}$ 
antigen, rather than unmodified $\mathrm{T}$ antigen, is essential for the normal localization of NMJ boutons. Moreover, glucuronylated core 1 glycans are also involved in NMJ arborization because $d C 1$ GalT1 and $d G l c A T-P$ mutants display a decreased number of NMJ branches on muscles 6 and 7.

Various ultrastructural defects are observed on both the pre- and postsynaptic sides of NMJ boutons in dC1GalT1 and dGlcAT-P mutants (Itoh et al., 2016, 2018). Although most of these defects differ between the two mutants, both show a decrease in the length of postsynaptic density (PSD), which comprises huge protein complexes including GluRs and scaffolding proteins. Therefore, these data suggest that mucin-type $O$-glycans synthesized by dC1GalT1 and dGlcAT-P are also involved in PSD formation.

\section{DISCUSSION}

Although little is known about their function in the mammalian nervous system, the physiological roles of mucin-type $O$-glycans have been well studied in this organ in Drosophila. Mucin-type O-glycans have been shown to be expressed in the Drosophila nervous system, including the CNS and NMJs. In primary cultured neurons, mucin-type core 1 glycans are localized in the proximal axonal segment by Shot-mediated trafficking. Phenotypic analyses have shown that the mucin-type $O$-glycans produced by dC1GalT1 and dGlcAT-P might be involved in CNS development and peripheral nerve elongation. At NMJs, the mucin-type O-glycans regulated by ppGalNAcT3 and ppGalNAcT35A control synaptic molecular assemblies, neurotransmission strength, and synaptic plasticity via integrin signaling. Moreover, glucuronylated core 1 glycans contribute to normal NMJ bouton localization, NMJ arborization, and PSD formation. Thus, mucin-type $O$-glycans play crucial roles in the Drosophila nervous system. Because glucuronylated core 1 glycans are thought to correspond to mammalian sialylated core 1 glycans, as described above, we suggest the possibility that functions may be conserved between these two glycan structures in the nervous system of Drosophila and mammals, respectively.

A previous study has revealed that the glycoprotein fasciclin I (Fas I), a homophilic cell adhesion molecule, controls NMJ arborization and synaptic transmission (Zhong and Shanley, 1995), suggesting that cell adhesion between neuron and muscle regulates NMJ morphology and synaptic function. Overexpression of Fas I leads to altered morphology of muscle 6/7 NMJs, similar to that observed in dC1GalT1 and dGlcAT-P

\section{REFERENCES}

Akasaka-Manya, K., Kawamura, M., Tsumoto, H., Saito, Y., Tachida, Y., Kitazume, S., et al. (2017). Excess APP O-glycosylation by GalNAc-T6 decreases A $\beta$ production. J. Biochem. 161, 99-111. doi: 10.1093/jb/ mvw056

Aoki, K., Porterfield, M., Lee, S. S., Dong, B., Nguyen, K., McGlamry, K. H., et al. (2008). The diversity of O-linked glycans expressed during Drosophila melanogaster development reflects stage- and tissue-specific requirements for cell signaling. J. Biol. Chem. 283, 30385-30400. doi: 10.1074/jbc.M804 925200 mutants. In the glomerular epithelium of the mammalian kidney, Podocalyxin carrying negatively charged sialylated mucin-type $O$-gycans has been shown to have anti-adhesive properties, which is required for precise formation of the filtration slit (Takeda et al., 2000; Doyonnas et al., 2001). Negatively charged glucuronylated core 1 glycans in Drosophila may also have similar anti-adhesive properties. Therefore, we propose the idea that, in $d C 1 G a l T 1$ and $d G l c A T-P$ mutants, a decrease in glucuronylated core 1 glycans on cell adhesion molecules such as Fas I may facilitate neuron muscle interaction in NMJs and thus cause defects in NMJ arborization and localization.

Previous studies have revealed that ppGalNAcT13 is specifically expressed at a high level in the mouse brain and is essential for the differentiation of neural stem cells through glycosylation and stabilization of podoplanin (Zhang et al., 2003; Xu et al., 2016). Although information about the roles of mucin-type $O$-glycans in the mammalian brain remains limited, recent studies have suggested that mucin-type $O$-glycosylation by several ppGalNAcTs is associated with human brain disorders, including Alzheimer's disease (Akasaka-Manya et al., 2017; Liu et al., 2017) and a congenital disorder of glycosylation (CDG; Zilmer et al., 2020). Because the basic structures of mucin-type O-glycans, including $\mathrm{Tn}$ antigen and $\mathrm{T}$ antigen, are conserved between Drosophila and mammals, the findings in Drosophila nervous system will be helpful in our understanding of not only the roles of mucin-type $O$-glycans in the mammalian brain but also the mechanisms underlying human brain disorders.

Drosophila is also used as a model system for studying complex brain functions, including cognitive behavior, learning, and sleep. In future studies, analyzing the impact of glycosylation deficiency in these brain functions is likely to lead to the discovery of unprecedented glycan functions in the mammalian brain.

\section{AUTHOR CONTRIBUTIONS}

All authors listed have edited and revised this manuscript. All authors contributed to the article and approved the submitted version.

\section{FUNDING}

This work was partially supported by Japan Agency for Medical Research and Development (AMED) Grant Number 1009765 (to $\mathrm{SN}$ ).

Baker, R., Nakamura, N., Chandel, I., Howell, B., Lyalin, D., and Panin, V. M. (2018). Protein O-mannosyltransferases affect sensory axon wiring and dynamic chirality of body posture in the Drosophila embryo. J. Neurosci. 38, 1850-1865. doi: 10.1523/JNEUROSCI.0346 $-17.2017$

Bennett, E. P., Mandel, U., Clausen, H., Gerken, T. A., Fritz, T. A., and Tabak, L. A. (2012). Control of mucin-type O-glycosylation: a classification of the polypeptide GalNAc-transferase gene family. Glycobiology 22, 736-756. doi: $10.1093 /$ glycob/cwr182

Berger, E. G. (1999). Tn-syndrome. Biochim. Biophys. Acta 1455, 255-268. doi: $10.1016 /$ s0925-4439(99)00069-1 
Bogdanik, L., Framery, B., Frölich, A., Franco, B., Mornet, D., Bockaert, J., et al. (2008). Muscle dystroglycan organizes the postsynapse and regulates presynaptic neurotransmitter release at the Drosophila neuromuscular junction. PLoS One 3:e2084. doi: 10.1371/journal.pone.0002084

Boskovski, M. T., Yuan, S., Pedersen, N. B., Goth, C. K., Makova, S., Clausen, H., et al. (2013). The heterotaxy gene GALNT11 glycosylates Notch to orchestrate cilia type and laterality. Nature 504, 456-459. doi: 10.1038/nature12723

Breloy, I., Schwientek, T., Althoff, D., Holz, M., Koppen, T., Krupa, A., et al. (2016). Functional analysis of the glucuronyltransferases GlcAT-P and GlcAT-S of Drosophila melanogaster: distinct activities towards the O-linked T-antigen. Biomolecules 6:8. doi: 10.3390/biom6010008

Breloy, I., Schwientek, T., Lehr, S., and Hanisch, F.-G. G. (2008). Glucuronic acid can extend O-linked core 1 glycans, but it contributes only weakly to the negative surface charge of Drosophila melanogaster schneider-2 cells. FEBS Lett. 582, 1593-1598. doi: 10.1016/j.febslet.2008.04.003

Dani, N., Zhu, H., and Broadie, K. (2014). Two protein N-acetylgalactosaminyl transferases regulate synaptic plasticity by activity-dependent regulation of integrin signaling. J. Neurosci. 34, 13047-13065. doi: 10.1523/JNEUROSCI. 1484-14.2014

Doyonnas, R., Kershaw, D., and Duhme, C. (2001). Anuria, omphalocele and perinatal lethality in mice lacking the CD34-related protein podocalyxin. J. Exp. Med. 194, 13-27. doi: 10.1084/jem.194.1.13

Fakhro, K. A., Choi, M., Ware, S. M., Belmont, J. W., Towbin, J. A., Lifton, R. P., et al. (2011). Rare copy number variations in congenital heart disease patients identify unique genes in left-right patterning. Proc. Natl. Acad. Sci. 108, 2915-2920. doi: 10.1073/pnas. 1019645108

Fuseya, S., Suzuki, R., Okada, R., Hagiwara, K., Sato, T., Narimatsu, H., et al. (2020). Mice lacking core 1-derived O-glycan in podocytes develop transient proteinuria, resulting in focal segmental glomerulosclerosis. Biochem. Biophys. Res. Commun. 523, 1007-1013. doi: 10.1016/j.bbrc.2020.01.033

Haines, N., Seabrooke, S., and Stewart, B. A. (2007). Dystroglycan and protein O-mannosyltransferases 1 and 2 are required to maintain integrity of Drosophila larval muscles. Mol. Biol. Cell 18, 4721-4730. doi: 10.1091/mbc.e0701-0047

Hiki, Y. (2009). O-linked oligosaccharides of the IgA1 hinge region: roles of its aberrant structure in the occurrence and/or progression of IgA nephropathy. Clin. Exp. Nephrol. 13, 415-423. doi: 10.1007/s10157-009-0173-7

Ichimiya, T., Manya, H., Ohmae, Y., Yoshida, H., Takahashi, K., Ueda, R., et al. (2004). The twisted abdomen phenotype of Drosophila POMT1 and POMT2 mutants coincides with their heterophilic protein O-mannosyltransferase activity. J. Biol. Chem. 279, 42638-42647. doi: 10.1074/jbc.M404900200

Itoh, K., Akimoto, Y., Fuwa, T. J., Sato, C., Komatsu, A., and Nishihara, S. (2016). Mucin-type core 1 glycans regulate the localization of neuromuscular junctions and establishment of muscle cell architecture in Drosophila. Dev. Biol. 412, 114-127. doi: 10.1016/j.ydbio.2016.01.032

Itoh, K., Akimoto, Y., Kondo, S., Ichimiya, T., Aoki, K., Tiemeyer, M., et al. (2018). Glucuronylated core 1 glycans are required for precise localization of neuromuscular junctions and normal formation of basement membranes on Drosophila muscles. Dev. Biol. 436, 108-124. doi: 10.1016/j.ydbio.2018. 02.017

Ju, T., and Cummings, R. D. (2002). A unique molecular chaperone Cosmc required for activity of the mammalian core $1 \beta 3$-galactosyltransferase. Proc. Natl. Acad. Sci. 99, 16613-16618. doi: 10.1073/pnas.262438199

Ju, T., and Cummings, R. D. (2005). Protein glycosylation: chaperone mutation in Tn syndrome. Nature 437:1252. doi: 10.1038/4371252a

Ju, T., Lanneau, G. S., Gautam, T., Wang, Y., Xia, B., Stowell, S. R., et al. (2008). Human tumor antigens $\mathrm{Tn}$ and sialyl Tn arise from mutations in cosmc. Cancer Res. 68, 1636-1646. doi: 10.1158/0008-5472.CAN-07-2345

Jumbo-Lucioni, P., Parkinson, W., and Broadie, K. (2014). Overelaborated synaptic architecture and reduced synaptomatrix glycosylation in a Drosophila classic galactosemia disease model. Dis. Model. Mech. 7, 1365-1378. doi: 10.1242/dmm.017137

Katsuki, T., Ailani, D., Hiramoto, M., and Hiromi, Y. (2009). Intra-axonal patterning: intrinsic compartmentalization of the axonal membrane in Drosophila neurons. Neuron 64, 188-199. doi: 10.1016/j.neuron.2009.08.019

Kim, B.-T. T., Tsuchida, K., Lincecum, J., Kitagawa, H., Bernfield, M., and Sugahara, K. (2003). Identification and characterization of three Drosophila melanogaster glucuronyltransferases responsible for the synthesis of the conserved glycosaminoglycan-protein linkage region of proteoglycans. Two novel homologs exhibit broad specificity toward oligosaccharides from proteoglycans, glycoproteins and glycosphingolipids. J. Biol. Chem. 278, 9116-9124. doi: 10.1074/jbc.M209344200

Kinoshita, T., Mori, Y., Hirano, K., Sugimoto, S., Okuda, K., Matsumoto, S., et al. (2014). Immuno-electron microscopy of primary cell cultures from genetically modified animals in liquid by atmospheric scanning electron microscopy. Microsc. Microanal. 20, 469-483. doi: 10.1017/S1431927614000178

Kinoshita, T., Sato, C., Fuwa, T. J., and Nishihara, S. (2017). Short stop mediates axonal compartmentalization of mucin-type core 1 glycans. Sci. Rep. 7:41455 doi: $10.1038 /$ srep41455

Koles, K., Irvine, K. D., and Panin, V. M. (2004). Functional characterization of Drosophila sialyltransferase. J. Biol. Chem. 279, 4346-4357. doi: 10.1074/jbc. M309912200

Koper, A., Schenck, A., and Prokop, A. (2012). Analysis of adhesion molecules and basement membrane contributions to synaptic adhesion at the Drosophila embryonic NMJ. PLoS One 7:e36339. doi: 10.1371/journal.pone.0036339

Kramerov, A. A., Arbatsky, N. P., Rozovsky, Y. M., Mikhaleva, E. A., Polesskaya, O. O., Gvozdev, V. A., et al. (1996). Mucin-type glycoprotein from Drosophila melanogaster embryonic cells: characterization of carbohydrate component. FEBS Lett. 378, 213-218. doi: 10.1016/0014-5793(95)01444-6

Kudo, T., Sato, T., Hagiwara, K., Kozuma, Y., Yamaguchi, T., Ikehara, Y., et al. (2013). C1galt1-deficient mice exhibit thrombocytopenia due to abnormal terminal differentiation of megakaryocytes. Blood 122, 1649-1657. doi: 10.1182/blood-2012-12-471102

Lin, Y., Reddy, B. V. V. G., and Irvine, K. D. (2008). Requirement for a core 1 galactosyltransferase in the Drosophila nervous system. Dev. Dyn. 237, 3703-3714. doi: 10.1002/dvdy.21775

Liu, F., Xu, K., Xu, Z., de las Rivas, M., Wang, C., Li, X., et al. (2017). The small molecule luteolin inhibits $\mathrm{N}$-acetyl- $\alpha$-galactosaminyltransferases and reduces mucin-type O-glycosylation of amyloid precursor protein. J. Biol. Chem. 292, 21304-21319. doi: 10.1074/jbc.M117.814202

Menon, K. P., Carrillo, R. A., and Zinn, K. (2013). Development and plasticity of the Drosophila larval neuromuscular junction. Wiley Interdiscip. Rev. Dev. Biol. 2, 647-670. doi: 10.1002/wdev.108

Müller, R., Hülsmeier, A. J., Altmann, F., Hagen, K. T., Tiemeyer, M., and Hennet, T. (2005). Characterization of mucin-type core-1 beta1-3 galactosyltransferase homologous enzymes in Drosophila melanogaster. FEBS J. 272, 4295-4305. doi: 10.1111/j.1742-4658.2005.04838.x

Nakamura, N., Stalnaker, S. H., Lyalin, D., Lavrova, O., Wells, L., and Panin, V. M. (2010). Drosophila dystroglycan is a target of O-mannosyltransferase activity of two protein O-mannosyltransferases, rotated abdomen and twisted. Glycobiology 20, 381-394. doi: 10.1093/glycob/cwp189

Nishihara, S. (2020). Functional analysis of glycosylation using Drosophila melanogaster. Glycoconj. J. 37, 1-14. doi: 10.1007/s10719-019-09892-0

Pandey, R., Blanco, J., and Udolph, G. (2011). The glucuronyltransferase GlcAT-P is required for stretch growth of peripheral nerves in Drosophila. PLoS One 6:e28106. doi: 10.1371/journal.pone.0028106

Pastor-Pareja, J. C., and Xu, T. (2011). Shaping cells and organs in Drosophila by opposing roles of fat body-secreted collagen IV and perlecan. Dev. cell 21, 245-256. doi: 10.1016/j.devcel.2011.06.026

Radhakrishnan, P., Dabelsteen, S., Madsen, F. B., Francavilla, C., Kopp, K. L., Steentoft, C., et al. (2014). Immature truncated O-glycophenotype of cancer directly induces oncogenic features. Proc. Natl. Acad. Sci. 111, E4066-E4075. doi: 10.1073/pnas.1406619111

Schneider, M., and Baumgartner, S. (2008). Differential expression of dystroglycan-spliceforms with and without the mucin-like domain during Drosophila embryogenesis. Fly (Austin) 2, 29-35. doi: 10.4161/fly.5726

Schwientek, T., Mandel, U., Roth, U., Müller, S., and Hanisch, F.-G. (2007). A serial lectin approach to the mucin-type O-glycoproteome of Drosophila melanogaster S2 cells. Proteomics 7, 3264-3277. doi: 10.1002/pmic.200600793

Springer, G. (1984). T and Tn, general carcinoma autoantigens. Science 224, 1198-1206. doi: 10.1126/science.6729450

Stotter, B. R., Talbot, B. E., Capen, D. E., Artelt, N., Zeng, J., Matsumoto, Y., et al. (2020). Cosmc-dependent mucin-type O-linked glycosylation is essential for podocyte function. Am. J. Physiol. Renal. Physiol. 318, F518-F530. doi: 10.1152/ajprenal.00399.2019

Suzuki, H., Moldoveanu, Z., Hall, S., Brown, R., Vu, H. L., Novak, L., et al. (2008). IgA1-secreting cell lines from patients with IgA nephropathy 
produce aberrantly glycosylated IgA1. J. Clin. Invest. 118, 629-639. doi: 10.1172/JCI33189

Takeda, T., Go, W., Orlando, R., and Farquhar, M. (2000). Expression of podocalyxin inhibits cell-cell adhesion and modifies junctional properties in Madin-Darby canine kidney cells. Mol. Biol. Cell 11, 3219-3232. doi: $10.1091 / \mathrm{mbc} .11 .9 .3219$

Tian, E., and Ten Hagen, K. G. (2007). O-linked glycan expression during Drosophila development. Glycobiology 17, 820-827. doi: 10.1093/glycob/cwm056

Tian, E., Hoffman, M. P., and Hagen, K. G. (2012). O-glycosylation modulates integrin and FGF signalling by influencing the secretion of basement membrane components. Nat. Commun. 3:869. doi: 10.1038/ncomms1874

Tran, D. T., and Hagen, K. G. (2013). Mucin-type O-Glycosylation during development. J. Biol. Chem. 288, 6921-6929. doi: 10.1074/jbc.R112.418558

Ueyama, M., Akimoto, Y., Ichimiya, T., Ueda, R., Kawakami, H., Aigaki, T., et al. (2010). Increased apoptosis of myoblasts in Drosophila model for the WalkerWarburg syndrome. PloS One 5:e11557. doi: 10.1371/journal.pone.0011557

Ugur, B., Chen, K., and Bellen, H. J. (2016). Drosophila tools and assays for the study of human diseases. Dis. Model. Mech. 9, 235-244. doi: 10.1242/dmm. 023762

Wairkar, Y. P., Fradkin, L. G., Noordermeer, J. N., and DiAntonio, A. (2008). Synaptic defects in a Drosophila model of congenital muscular dystrophy. J. Neurosci. 28, 3781-3789. doi: 10.1523/JNEUROSCI.0478-08.2008

Wang, Y., Jobe, S. M., Ding, X., Choo, H., Archer, D. R., Mi, R., et al. (2012). Platelet biogenesis and functions require correct protein O-glycosylation. Proc. Natl. Acad. Sci. U S A 109, 16143-16148. doi: 10.1073/pnas. 1208253109

Xu, Y., Pang, W., Lu, J., Shan, A., and Zhang, Y. (2016). Polypeptide $\mathrm{N}$-acetylgalactosaminyltransferase 13 contributes to neurogenesis via stabilizing the mucin-type O-glycoprotein podoplanin. J. Biol. Chem. 291, 23477-23488. doi: 10.1074/jbc.M116.743955

Yamamoto-Hino, M., Yoshida, H., Ichimiya, T., Sakamura, S., Maeda, M., Kimura, Y., et al. (2015). Phenotype-based clustering of glycosylationrelated genes by RNAi-mediated gene silencing. Genes Cells 20, 521-542. doi: $10.1111 /$ gtc. 12246
Yoshida, H., Fuwa, T. J., Arima, M., Hamamoto, H., Sasaki, N., Ichimiya, T., et al. (2008). Identification of the Drosophila core $1 \beta 1,3$-galactosyltransferase gene that synthesizes $\mathrm{T}$ antigen in the embryonic central nervous system and hemocytes. Glycobiology 18, 1094-1104. doi: 10.1093/glycob/cwn094

Zhang, Y., Iwasaki, H., Wang, H., Kudo, T., Kalka, T. B., Hennet, T., et al. (2003). Cloning and characterization of a new human UDP-N-acetyl- $\alpha-\mathrm{d}$ galactosamine: polypeptide $\mathrm{N}$-acetylgalactosaminyltransferase, designated ppGalNAc-T13, that is specifically expressed in neurons and synthesizes GalNAc $\alpha$-serine/threonine antigen*. J. Biol. Chem. 278, 573-584. doi: 10.1074/jbc. M203094200

Zhong, Y., and Shanley, J. (1995). Altered nerve terminal arborization and synaptic transmission in Drosophila mutants of cell adhesion molecule Fasciclin I. J. Neurosci. 15, 6679-6687. doi: 10.1523/JNEUROSCI.15-10-06679.1995

Zilmer, M., Edmondson, A. C., Khetarpal, S. A., Alesi, V., Zaki, M. S., Rostasy, K., et al. (2020). Novel congenital disorder of O-linked glycosylation caused by GALNT2 loss of function. Brain 143, 1114-1126. doi: 10.1093/brain/ awaa063

Conflict of Interest: The authors declare that the research was conducted in the absence of any commercial or financial relationships that could be construed as a potential conflict of interest.

Publisher's Note: All claims expressed in this article are solely those of the authors and do not necessarily represent those of their affiliated organizations, or those of the publisher, the editors and the reviewers. Any product that may be evaluated in this article, or claim that may be made by its manufacturer, is not guaranteed or endorsed by the publisher.

Copyright (c) 2021 Itoh and Nishihara. This is an open-access article distributed under the terms of the Creative Commons Attribution License (CC BY). The use, distribution or reproduction in other forums is permitted, provided the original author(s) and the copyright owner(s) are credited and that the original publication in this journal is cited, in accordance with accepted academic practice. No use, distribution or reproduction is permitted which does not comply with these terms. 